\title{
A Prediction Model for Spread of Ebola Virus
}

\author{
Zhuo Chen
}

School of North China Electric Power University, Baoding 071003, China;

953167915@qq.com

Keywords: Ebola; spread;SIR

Abstract. The 2014 Ebola outbreak is an ongoing epidemic of the Ebola virus disease in West Africa causing many deaths and great panic. It has been declared "the largest, most complex and most severe we've ever seen” by the World Health Organization (WHO) ${ }^{[1]}$.

This paper tries to establish a realistic, sensible, and useful model to optimize the eradication of Ebola. First, we establish a SIR (Susceptible-Infective-Removed) model ${ }^{[2]}$ to reveal the natural spread process of the Ebola disease in the crowd. Then we select three countries- Guinea, Sierra Leone and Libya, where the epidemic disease is serious, to test the model. The simulation result fits well with the true data.

\section{Introduction}

\subsection{Restatement of the problem}

The 2014 Ebola Outbreak is an ongoing epidemic of the Ebola virus(EBOV) disease in West Africa that first began in Guinea in March 2014. Following its subsequent spread to Liberia, Sierra Leone and Nigeria, which has claimed the lives of at least 3,100 patients since the initial outbreak, the Ebola epidemic has been declared "the largest, most complex and most severe we've ever seen" by the World Health Organization (WHO) and major news outlets around the world ${ }^{[3]}$.

The Ebola virus can cause a severe disease, it is a fatal infectious diseases without treatment and popular in western Africa like Guinea, Sierra Leone and Liberia. Fig $1{ }^{[4]}$ shows the main region affected by the virus in west Africa. Considering the huge global influence of Ebola, it's an urgent task to think out an approach to eradicate its spread. Fortunately, the world medical association has announced that their new medication could stop Ebola and cure patients whose disease is not advanced. For a better treatment, we have to realise the situation of transmission of the virus. Therefore, a realistic, sensible, and useful model is needed to help to predict and eradicate the virus.

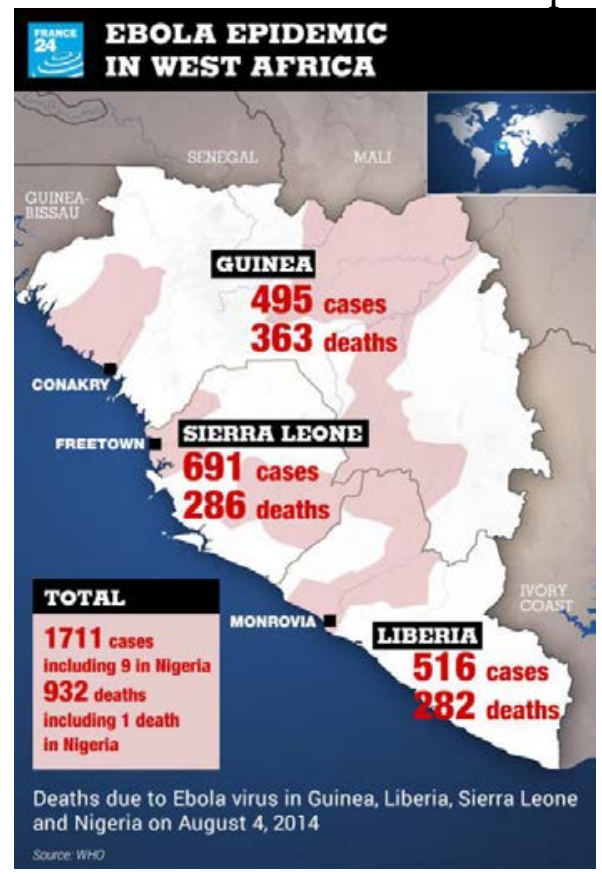

Figure1 Ebola epidemic in west Africa 


\subsection{Our work}

we establish a SIR (Susceptible-Infective-Removed) model to reveal the natural spread process of the Ebola disease in the crowd. Then we select three countries- Guinea, Sierra Leone and Libya, where the epidemic disease is serious, to test the model.

\section{Assumptions}

- There is no difference between the individual in groups except those who are infectious. Patients and healthy people are homogeneously mixed in the crowed.

- The number of people,being observed, is large enough. Considering only the average effect of transmission process of EBOV.

- The chance that a healthy person is infected is proportional to the chance that he can contact with the patients.

- The process of birth and death is neglected, and the immigration and emigration of people is also ignored. That is,the population of the region we study is a constant.

- All the medical centers are built in the provincial capitals of the regions in a country.

\section{Symbols}

Table 1 Symbols for model SIR

\begin{tabular}{c|c}
\hline symbols & definitions \\
\hline $\boldsymbol{s}$ & susceptible individuals \\
$\boldsymbol{r}$ & infectious individuals \\
$\boldsymbol{k}$ & recover and dead individuals \\
$\boldsymbol{h}$ & the transmission rate of EBOV \\
\hline
\end{tabular}

\section{The model}

\section{1 establishing the model (SIR)}

Suppose the patients (infectious individuals) spread the germs to healthy people (susceptible individuals) through the air, food, etc. The number of healthy people that a patient can infect in unit time is a constant $\boldsymbol{k}$. The patients can be cured at a fixed rate, $\boldsymbol{h}$, and become immune to the disease .The other patients who don't get effective treatment finally die. These two kinds of people are regarded as a new kind, the recover and dead individuals. Fig 2 shows the process.

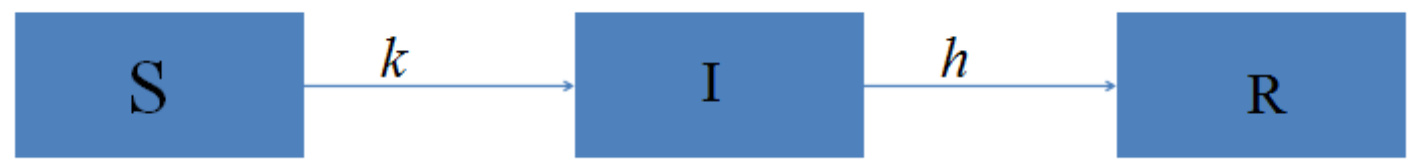

Figure 2 the process of transmission of illness

The number of healthy people, patients, recover and dead individuals at time $t$ is $s(t), i(t)$ and $r(t)$ respectively. We assume they are all continuously differentiable functions about time $\boldsymbol{t}$. According to the assumption, we can get the following formula, 


$$
\begin{aligned}
i(t+\Delta t)-i(t) & =k i(t) s(t) \Delta t-h i(t) \Delta t, \quad \text { or } \\
\frac{d i}{d t} & =k i(t) s(t)-h i(t) .
\end{aligned}
$$

Similarly, we have

$$
\begin{aligned}
& \frac{d s}{d t}=-k i(t) s(t) . \\
& \frac{d r}{d t}=h i(t) .
\end{aligned}
$$

\section{2 testing the model}

Based on the program we devise, We simulate the change trend of the number of the diseased in the three countries. We obtain some figures which can visually reveal the tendency.

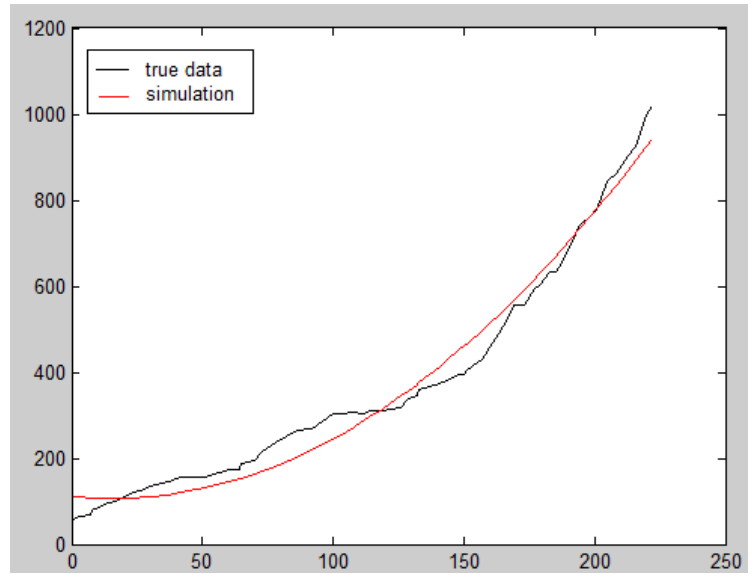

Figure 3 The spread of Ebola of Guinea

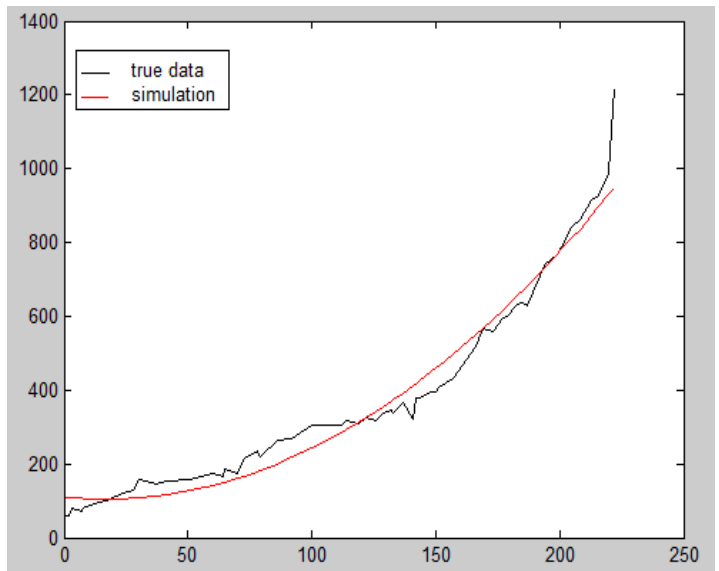

Figure 4 The spread of Ebola of Sierra Leone

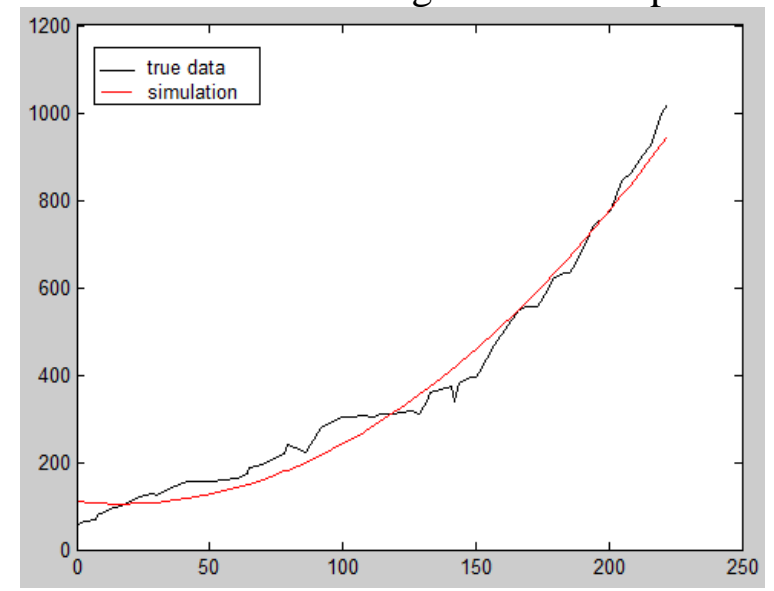

Figure 5 The spread of Ebola of Libya

\section{Conclusion}

The Ebola epidemic is continue to rise in 2014 which warns the people all over the world constantly. The outbreaks in Guinea, Sierra Leone and Liberia are especially serious. It is high time that we should accelerate the pace of containing the diffusion of the epidemic.Therefore, we begin by researching the spread of the Ebola virus, and establish the model to predict the increase of the number of infectious people in the three countries respectively.According to the result of the model testing, the prediction model is successful. 


\section{References}

[1]http://www.who.int/zh/

[2]http://www.docin.com/p-19555100.html

[3]http://www.islandvoice.org/ebola-update-january-2015/

[4] http://knowyourmeme.com/memes/events/2014-ebola-outbreak 